\title{
A Genetic-algorithm-based Weighted Clustering Algorithm in MANET
}

\author{
Hua Yang ${ }^{1}$ and Zhi-mei $\mathrm{Li}^{2}$ \\ ${ }^{1}$ Guilin University of Aerospace Technology, Guilin, China \\ ${ }^{2}$ Guilin University of Aerospace Technology, Guilin, China \\ 'yanghua_719@163.com, ${ }^{2}$ zhimeili2006@163.com
}

\begin{abstract}
Weighted clustering algorithm (WCA) is an important Clustering Algorithm in mobile ad hoc network (MANET). This paper conducts the research on WCA algorithm and nodes parameters, and introduces GA-WCA based on improving WCA by Genetic algorithm. GA-WCA is able to quickly potion cluster head node of the grouping and forms the stable clustering set between nodes, which will improve the validity and stability of MANET. This paper simulates on GA-WCA by NS3, the result indicates that GA-WAC is more effectively to form stable grouping than WCA, lowers the information updating expenses of the grouping network, and optimizes the usage of network bandwidth. GAWCA effectively improves the MANET availability and survivability.
\end{abstract}

Keywords: Clustering, Genetic algorithm, MANET, WCA

\section{Introduction}

The architecture of mobile ad hoc network can be flat or hierarchical. For the MANET with flat architecture, function and status of all nodes in network are the same and its biggest advantage is that structure is simple and source node can communicate with destination node as long as there is route between them. However, flat MANET has poor scalability. Some papers [1-2] pointed out even in an ideal environment, the throughput of each node still decrease with the increasing number of nodes in network. But the using of clustering is a good method to extend MANET. Hierarchical network architecture is capable of dynamically adapt the change of network structure. The head node within a group is responsible for establishing cluster and maintaining network topology, as well as make resource allocation for other nodes within the same cluster. Due to the mobility of nodes in MANET, joining cluster or leaving cluster of a node will both affect the stability of the cluster, but at the same time, frequent changes of head node will affect protocol performance that reply on head node. Therefore, clustering MANET is the main problem for designing hierarchical network architecture.

In related research of MANET, research on clustering algorithm in hierarchical MANET is a very important and cutting-edge issue. Many universities and research institutions continue to do this challenging research project. As far as the currently disclosed literature and technical information, main clustering algorithms in MANET are LCA [3], MinID [4-5], MAX-Degree [6-7], DCA [8-10], WCA [11-14] and so on. LCA is a groundbreaking clustering algorithm proposed by Baker et al., and it is a clustering algorithm for high frequency communication network of task force. LCA can conveniently construct clustering structure, but will produce excessive cluster head nodes, especially when the node ID is linear ascending order. MinID proposed by Grela and Tsai made some improvements to LCA algorithm and further reduced the number of head nodes. Calculation amount of this clustering algorithm is small and easy to implement. In addition, its convergence speed is also fast. Update frequency of head nodes in this algorithm is slow and spending to maintain this cluster is small. However, the 
disadvantage of this algorithm is that it is prone to choose those nodes with smaller ID as head nodes, resulting in these nodes consume more battery power, thus shortening the time that the entire network begin to segment. In addition, this algorithm also did not consider factors such as load balancing. MAX-Degree referred the methods to choose router in Internet and chose those nodes with highest degree (having most neighboring nodes) as head nodes. When degree is the same, choose the nodes with smallest ID as head nodes, the first hop node of head node becomes ordinary member node of this cluster. Although this algorithm can reduce the number of clusters and the transfer delay of packet, because the algorithm does not limit the number of nodes in cluster, when the number of nodes is excessive, throughput of each customer node will decrease sharply. In addition, when the mobility of node is strong, the update frequency of head nodes will increase sharply, resulting in huge maintenance cost. Distributed Clustering Algorithm (DCA) is similar to MinID, but DCA chooses head node according to the weights of nodes, which means the greater the weight of node is, the higher priority of node is. With this algorithm, head node can be uniformly dispersed in various parts of the network, but due to the periodicity of algorithm execution, single mapping of weights, nodes cannot move especially during the execution of the algorithm and low adaptation to mobility, all those defects limit the application environment and scale of this algorithm. WCA is an ondemand distributed generation clustering algorithm. Electoral process of WCA clustering algorithm is not periodical and update frequency is low, thus it can maintain the stability of network and reduce the cost of computation and communication. In WCA, when all the head nodes that form extreme cases form a linear plot, it cannot search multiple cluster parallel and thus route searching time will increase linearly, because communication between cluster can only search one cluster when search destination node. Because WCA has wide application in hierarchy architecture in MANET, this paper retained the advantage of WCA and used genetic algorithm to search head nodes parallel and revised WCA to GA-WCA, considering the disadvantage of WCA.

\section{Weighted Clustering Algorithm}

WCA is an on-demand, distributed, weight-based MANET clustering algorithm. It considered ideal node density, battery power and its combination effect, flexible allocation and its weight factor of nodes when choosing head nodes. WCA can dynamically adapt to the continually change of MANET topology and limit the number of member nodes that head nodes deal with, which makes MAC function not decline. WCA will be executed only when needed, which means when node is neither head node nor belongs to any cluster, WCA will be executed, in order to reduce the computation and communication cost.

The process of choosing head node in WCA is as following:

Step 1: Search the neighboring nodes of each node $V$ (nodes within its transmission coverage). The number of neighboring nodes of node $V$ is defined as $V$, density $d_{V}$,

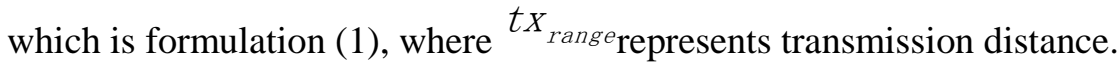

$$
d_{V}=|N(v)|=\sum_{V^{\prime} \in V, v^{\prime} \neq V}\left\{\operatorname{dist}\left(V^{\prime}, v\right)<t_{\text {range }}\right\}
$$

Step 2: Calculate the density difference $\Delta_{V}$ of each node $V$.

$$
\Delta_{V}=\left|d_{V}-\delta\right|
$$

Step 3: Calculate the sum of distance between each node $V$ and its all neighboring nodes.

$$
D_{v}=\sum_{v^{\prime} \in N(v)}\left\{\operatorname{dist}\left(v, v^{\prime}\right)\right\}
$$


Step 4: Calculate the average moving speed $M_{V}$ of each node $\mathrm{v}$ till current time $\mathrm{T}$, where $\left(X_{t}, Y_{t}\right)$ and $\left(X_{t-1}, Y_{t-1}\right)$ represent the position coordinates of node $\mathrm{v}$ at time $(t)$ and $(t-1)$ respectively. $M_{v}$ is the quantitative measure of node mobility.

$$
M_{V}=\frac{1}{T} \sum_{i=1}^{T} \sqrt{\left(X_{t}-X_{t-1}\right)^{2}+\left(Y_{t}-Y_{t-1}\right)^{2}}
$$

Step 5: Calculate the cumulative time $P_{v}$ of node $V$ as head node. $P_{v}$ represents the consuming battery power of node $V$. Assuming the battery power of head node is more than ordinary node's.

Step 6: Calculate the combination weight $W_{V}$ if each node $V$, where $W_{1},{ }^{W_{2}},{ }^{W_{3}},{ }^{W_{4}}$ represent node density, distance, mobility and weight factor of battery power respectively.

$$
W_{V}=W_{1} \Delta_{v}+W_{2} D_{V}+W_{3} M_{V}+W_{4} P_{V}
$$

Step 7: Choose the node with smallest combination weight as head node. The nodes that are head node's neighboring node withdraw from the election of head node.

Step 8: repeat Step 2 to 7, cluster the nodes that are neither head node nor in any cluster in network.

\section{Genetic-Algorithm-Based Weighted Clustering Algorithm}

Genetic algorithm is recognized as one of the effective method to solve NP problems and is widely applied in combinatorial optimization, machine learning, signal processing, self-adaptive control and artificial life, and made many remarkable achievements. Applying genetic algorithm into MANET to optimize clustering can achieve better results.

In order to improve the searching speed to head node of this algorithm, before the formal formation of cluster, build a virtual cluster from a certain node and its neighboring nodes within two hop and head node is not decided. In clustering process of GA-WCA, use genetic algorithm to search in a virtual cluster and at the same time, also hope that this algorithm can converge as soon as possible in order to save the battery energy of node and improve the surviving ability of clustering. Therefore, basic genetic algorithm has to adapt and improve to meet these objectives.

\subsection{Genetic Algorithm Process}

Initial cluster: Initial population of traditional genetic algorithm is randomly generated, and thus the quality of initial population is random, which is anachronistic in the clustering of MANET. The initial population is based on virtual clustering and randomly generated an initial individual in initial population and in this way it can ensure the difference between individuals and evenly distributed in the solution space, which improved the possibility of searching the global optimal that converge and the calculation amount is not that much.

Random Genetic Inheritance Strategy: In genetic manipulation, it may cause the loss of good genes, resulting in solving process becomes slow or fall into local optimum, and thus making the calculation amount of node too excessive and lead to excessive energy consumption, which is not conductive to the stability of network. To overcome such drawbacks, in the evolutionary process, it need to replace the bad individual with good one, in order to reduce the loss of good genes. In actual operation, choose two worst individuals in each generation and use the best individual of current generation to the best individual so far to replace them. In order to prevent the destruction of causal factors in 
selection, crossover and mutation from leading to loss of best individuals of parent generation in the next generation, it also uses the individual preservation method, which is not selecting and crossover the individuals with highest adaptability in parent generation and directly copied to the next generation. This selection method not only ensures the breadth of offspring on the solution spatial distribution, but also the adaptability of offspring, thus can greatly improve the operating efficiency of genetic algorithm.

The adaptive adjustment of crossover probability and mutation probability: The selfadaptability of crossover possibility $P_{c}$ and mutation possibility $P_{m}$ is also one of the effective means to improve the searching efficiency of genetic algorithm. Used adaption formulation of crossover possibility and mutation possibility is as following:

$$
\begin{aligned}
& P_{c}= \begin{cases}P_{c 1}-\frac{\left(P_{c 1}-P_{c 2}\right) \bullet\left(f^{\prime}-f_{a v g}\right)}{f_{\max }-f_{a v g}} & f^{\prime} \geq f_{a v g} \\
P_{c 1} & f^{\prime}<f_{a v g}\end{cases} \\
& P_{m}= \begin{cases}P_{m 1}-\frac{\left(P_{m 1}-P_{m 2}\right) \bullet\left(f^{\prime}-f_{a v g}\right)}{f_{\max }-f_{a v g}} & f \geq f_{a v g} \\
P_{m 1} & f<f_{a v g}\end{cases}
\end{aligned}
$$

In the formulation, $P_{c 1}=0.9, P_{c 2}=0.6, P_{m 1}=0.1, P_{m 2}=0.01, f_{\max \text { and }} f_{a v g}$ represent the biggest adaptability and average adaptability in each generation respectively. $f^{\prime}$ is the bigger adaptability in parent generation before crossover operation and $f$ is the adaptability of mutation individual. After adjustment, reduce the $P_{c}$ and $P_{m}$ of highquality individual and make the algorithm converge as quickly as possible. In addition, improving the adaptability that are lower than average adaptability can prevent algorithm from falling into local optimum.

Improvements to adaptability function: Average adaptability of population $f_{\text {avg }}$ and variance $\sigma$ are defined respectively:

$$
\begin{gathered}
f_{\text {avg }}=\sum_{i=1}^{N} f_{i} / N \\
\sigma=\sqrt{\sum_{i=1}^{N}\left(f_{i}-f_{a v g}\right)^{2} / N}
\end{gathered}
$$

And adjust the individual adaptability:

$$
f_{i}^{\prime}=f_{i}+\left(f_{\text {avg }}-c \bullet \sigma\right)
$$

where $f_{i}$ is the adaptability of individual $i, N$ is the population scale, $f_{i}^{\prime}$ is the adaptability of adjusted individual $i, c=2$.

If adjusted individual adaptability is smaller than 0 , then make it zero. Adaptability is a measure of the pros and cons of individual and above adjustment reduced the selection probability of calculating early privileged individuals and avoid the prematurity because of the excessive number of privileged individuals.

Termination Condition: In order to avoid excessive energy consumption of nodes, where taking the largest hereditary algebra $T$ and the best individual fitness value to continuously maintain to retain the algebraic $T^{\prime}$ is judged by a combination of evolutionary conditions of termination. Search for optimal solutions within a given hereditary algebra limited range, and determine whether the solution after several iterations later still the best, then launch evolution, or continue to search until the best 
individual to meet the minimum retention algebra. If the maximum genetic algebra, the direct output current optimal solution obtain.

\subsection{The Basic Describe of the Algorithm}

Algorithm implementation process is divided into two steps: initialization process and the clustering process. Initialization process clustering provides the necessary support parameters to genetic algorithm. The main steps are as follows:

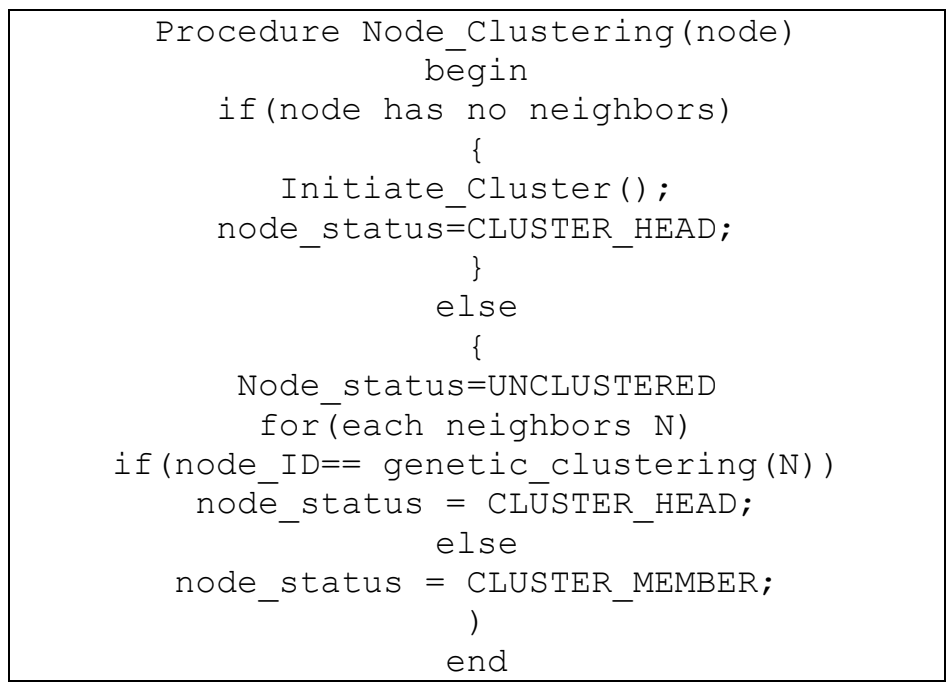

Figure 1. GAC Pseudo-Code

Initialization process : Switch node ID among nodes, the average velocity magnitude $\mathrm{v}$, the direction of velocity evaluation, the node position coordinates $(x, y)$, through the exchange of information, nodes can get neighbor nodes set their $N$, according to the formula (1) - (5) to get some basic parameters, initialize to provide the genetic algorithm clustering.

Clustering process: Use a Formula as the objective function, optimal returns based on genetic algorithm $\mathrm{CH}$ node, if the node ID is the equivalent of the best group $\mathrm{CH}$ node, the node is a cluster head node, the other nodes are CM nodes. Figure 1 shows the pseudo-code node clustering based on genetic algorithm.

$$
\operatorname{Min}\left(W_{V}\right)=W_{1} \Delta_{V}+W_{2} D_{V}+W_{3} M_{V}+W_{4} P_{V}
$$

Clustering Maintenance: After the completion of clustering, MANET topology is changing, node leave and joint will bring change of clustering structure, every change of clustering structure leads to the cost of computing and communication, in this respect, need to design a strategy to reduce the clustering change to maintain clustering. Node by periodically "hello" control message updates the neighbor node table, and set a time limit $\mathrm{T}$, the group is not received within the adjacent node "hello" message within the time $\mathrm{T}$, it shows that it has become isolated nodes of the cluster, If this node is CM node, then try to join an existing group, If it cannot join the group, then establish their own cluster; if this node is $\mathrm{CH}$ node, then start genetic algorithm to re-clustering.

\section{Simulation and Discussion}

\subsection{Performance Indicator}

You can follow a variety of measures to evaluate the genetic algorithm clustering strategy. Measuring is roughly divided into two different types, one is measuring the 
dynamic response characteristics of clustering algorithms, such as clustering stability, efficiency agreement; the second is to measure the performance of the packet level, such as latency and throughput. The way to test the effectiveness of genetic clustering algorithm through simulation, using the average performance evaluation. The average performance including the average size of nodes, the probability of participating clustering nodes, the average residence time in a given cluster, the average cluster survival time, the processing rate of controlling message of each node.

\subsection{Experimental Parameter Setting}

Table 1. Parameter Setting

\begin{tabular}{|l|l|}
\hline Parameters & Value \\
\hline Simulation range & $100 m \times 100 m$ \\
\hline Number of Nodes & Fixed at 200 \\
\hline Motion Model & Random walk Mobility Model \\
\hline Data Transmision rate & $1 \mathrm{Mbps}$ \\
\hline Wireless channel bandwidth & $11 \mathrm{Mbps}$ \\
\hline Data channel packet size & 512 Bytes \\
\hline Control channel packet size & 128 Bytes \\
\hline Transmission range & $0 \sim 80$ \\
\hline Movement speed Max_Disp & $1 \sim 10$ \\
\hline
\end{tabular}

Based on the above discussion, develop a simulation model to assess the stability and efficiency of genetic clustering algorithm. Simulation model construct a MANET model, simulate to genetic clustering algorithm based on NS3, simulation parameters as shown in Table I. After the simulation begins, immediately move all nodes in random Ad Hoc mobility model. The average speed of the mobile node is $5.0 \sim 25.0 \mathrm{Km} / \mathrm{h}$, the scene range of simulation node is set to $1000 \mathrm{~m} * 1000 \mathrm{~m}$, in the time frame, the moving speed of each node is normally distributed, the direction is random, each node in the random time change its moving direction and moving speed. Each simulation, within 1 hour observation time, one sample per second network status, collect experimental data. To exclude the effect of the moment, every two hours beginning of the experiment does not collect data.

\subsection{Simulation Results}

As shown is Figure 2, the average number of nodes as the $\mathrm{CH}$ transmission distance increases and decreases, as the WCA does not limit the size of the group, so that a sharp increase in the load of the cluster head in a relatively large radius, and GA-WCA group size is not restricted, but the first node of each cluster is the strongest overall performance in nodes, therefore, it can achieve an ideal result. With increasing radius, reducing delete nodes, the rule set update rate decreases, GA-WCA algorithm can enhance the stability of the cluster. 


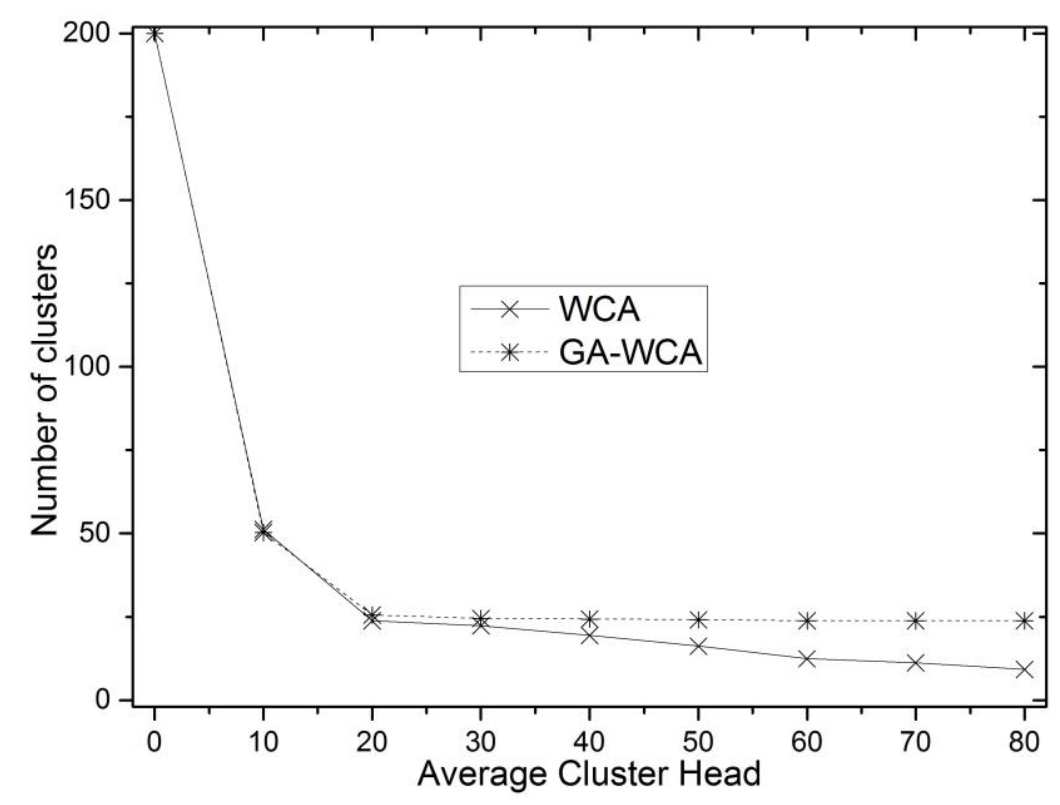

Figure 2. Number of Clusters

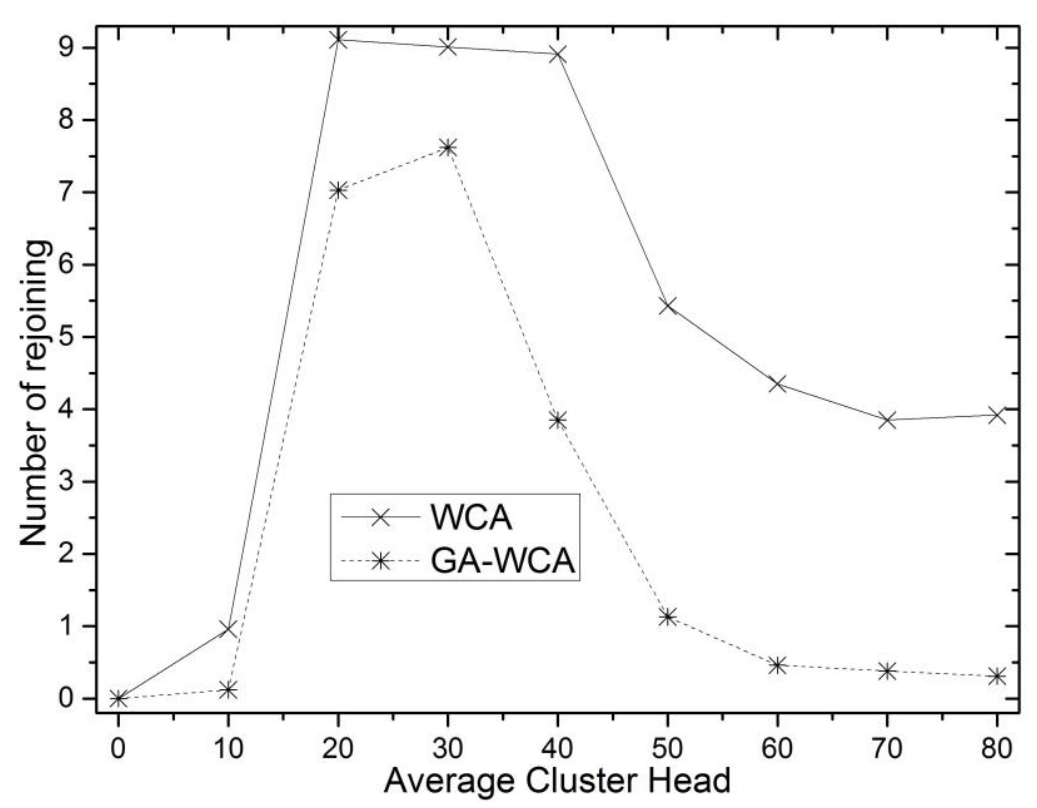

Figure 3. Number of Rejoining 


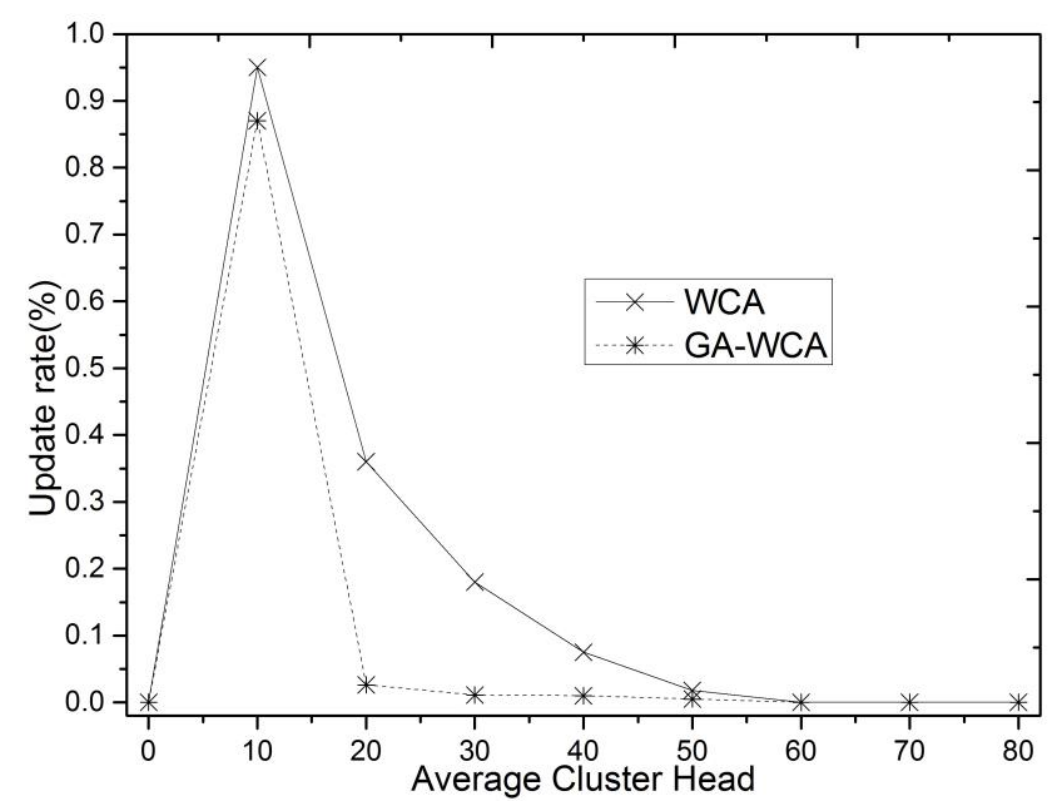

Figure 4. Cluster Update Rate

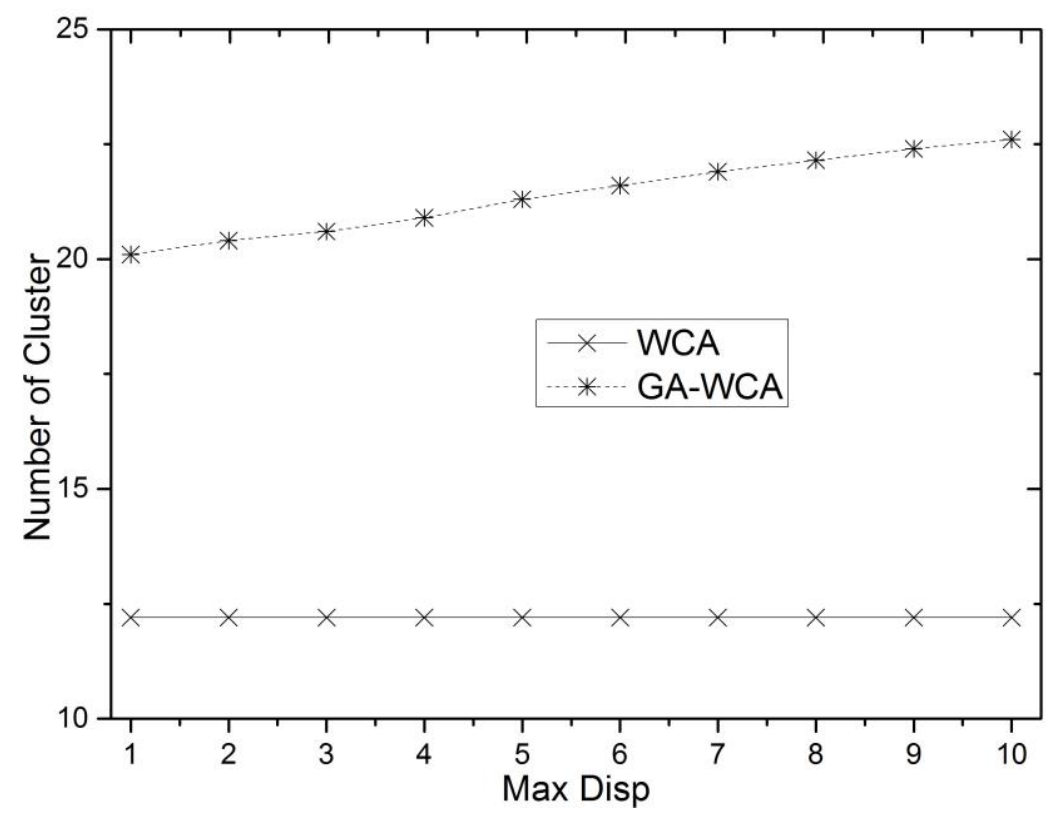

Figure 5. Number of Clusters

Figure 3, Figure 4 show a comparison of clustering stability, the radius increases, the node delete reduces, the rule set update rate decreases, GA-WCA algorithm can enhance the stability of the cluster. 


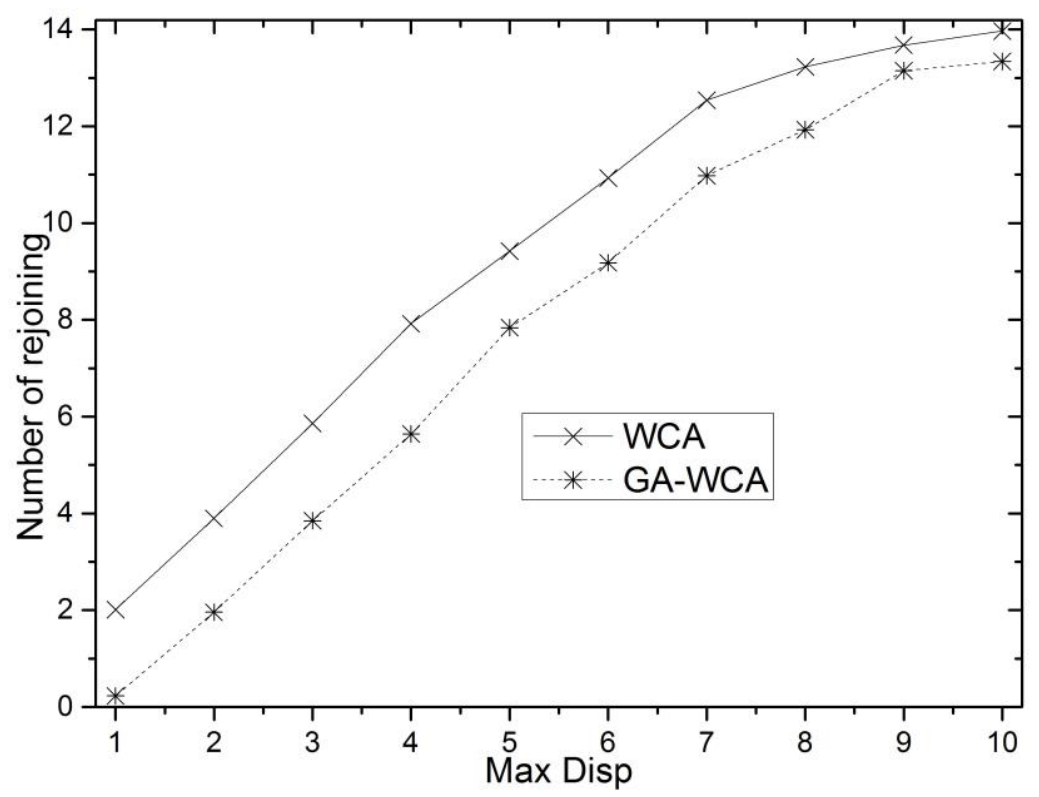

Figure 6. Number of Rejoining

\section{Conclusions}

Clustering structure is a good choice for massive MANET structure to further improve the performance of MANET. So it is necessary to quickly cluster the network nodes, keep the stability of the clustering structure, and reduce the cost of maintenance clustering and network routing in this structure. For the above objectives, this paper proposes a clustering algorithm based on genetic algorithm. GA-WCA conducts clustering optimization from MANET and optimizes by combinating $\mathrm{CH}$ power into the algorithm. The simulation result indicates that GA-WCA is based on WCA, but is prior to it.

\section{References}

[1] F. Shi, W.-J. Liu, D.-X. Jin and J.-S. Song, "A cluster-based countermeasure against blackhole attacks in MANETs", Journal of Telecommunication Systems, vol. 57, no. 2, (2014), pp. 119-136.

[2] O. V. Drugan, T. Plagemann and E. Munthe-Kaas, "Dynamic clustering in Sparse MANETs", Journal of Computer Communications, vol. 59, (2015), pp. 84-97.

[3] S. K. Gupta, P. Khatri and P. Agrawal, "Cost Based Scalable Clustering in MANET", Proceedings of the International Conference on Computational Intelligence and Communication Networks, (2014); Bhopal, India.

[4] P. Makani, S. Srivastava and S. Gupta, "Protocols Governing Clustering in MANETS", International Journal of Advanced Research in Computer Science, vol. 5, no. 3, (2014), pp. 202-207.

[5] M. Mehta, I. J. Rajput and V. Kumar Gupta, "A Survey of Clustering Approaches for Mobile Ad Hoc Network", Journal of Compusoft, vol. 3, no. 2 (2014), pp. 507.

[6] S. Aruna and A. Subramani, "Weighted Double Cluster Head Based Approach for Enhancing Route Stability in MANETS", Asian Journal of Information Technology. vol. 13, no. 11, (2014), pp. 725-732.

[7] B. Hassanabadi, C. Shea, L. Zhang and S. Valaee, "Clustering in vehicular ad hoc networks using affinity propagation", Journal of Ad Hoc Networks, no. 13, (2014), pp. 535-548.

[8] B. Muthusenthil and S. Murugavalli, "Location Aided Cluster Based Geographical Routing Protocol for Intermittently Connected MANET", Journal of International Review on Computers and Software, vol. 9, no. 1, (2014), pp. 1-8.

[9] D. J. Baker and A. Ephremides, "The architectural organization of a mobile radio network via a distributed algorithm", Journal of IEEE Transactions on Communication, vol. 29, no. 11, (1981), pp. 1694-1701.

[10] A. Ephremides, J. E. Wieselthier and D. J. Baker, "A design concept for reliable mobile radio networks with frequency hopping signaling", Proceedings of the IEEE, (1987).

[11] C. R. Lin and M. Gerla, "Adaptive clustering for mobile wireless networks", IEEE Journal on Selected areas in Communications, vol. 15, no. 7, (1997), pp. 1265-1275. 
[12] M. Gerla and J. T. C. Tsai, "Multicluster, mobile, multimedia radio network", Journal of Wireless networks, vol. 1, no. 3, (1995), pp. 255-265.

[13] A. K. Parekh, "Selecting routers in ad-hoc wireless networks", Proceedings SBT/IEEE Intl Telecommunications Symposium, (1994).

[14] S. Basagni, "Distributed clustering for ad hoc networks", In Parallel Architectures, Algorithms, and Networks, (1999), pp. 310-315.

[15] O. Younis and S. Fahmy, "HEED: a hybrid, energy-efficient, distributed clustering approach for ad hoc sensor networks", Journal of IEEE Transactions on Mobile Computing, vol. 3, no. 4, (2004), pp. 366379.

[16] O. Younis and S. Fahmy, "Distributed clustering in ad-hoc sensor networks: A hybrid, energy-efficient approach", Proceedings of the Twenty-third AnnualJoint Conference of the IEEE Computer and Communications Societies, (2004); HongKong, China.

[17] M. Chatterjee, S. K. Das and D. Turgut, "WCA: A weighted clustering algorithm for mobile ad hoc networks", Journal of Cluster Computing, vol. 5, no. 2, (2002), pp. 193-204.

[18] W. Choi and M. Woo, "A distributed weighted clustering algorithm for mobile ad hoc networks", Proceedings of the Advanced International Conference on Telecommunications and International Conference on Internet and Web Applications and Services, (2006); Guadeloupe, French Caribbean.

[19] H. Jia, S. Ding, H. Zhu, F. Wu and L. Bao, "A feature weighted spectral clustering algorithm based on knowledge entropy”, Journal of Software, vol. 8, no. 5, (2013), pp. 1101-1108.

[20] K. Xia, Y. Wu, X. Ren and Y. Jin, "Research in Clustering Algorithm for Diseases Analysis", Journal of Networks, vol. 8, no. 7, (2013), pp. 1632-1639.

[21] P. Gupta and P. R. Kumar, " The capacity of wireless networks", Journal of IEEE Transactions on Information Theory, vol. 46, no. 2, (2000), pp. 388-404.

[22] K. Xu, X. Hong and M. Gerla, "An ad hoc network with mobile backbones", Proceedings of the IEEE International Conference on Communication, (2002); New York, USA.

\section{Authors}
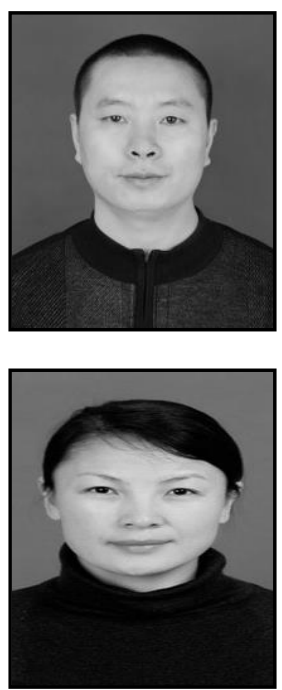

Hua Yang, He received M. S. degree in Computer Science from Guangxi normal university, Guilin, China, in 2011.He is currently associate professor of Guilin university of Aerospace Technology, Guilin, China. His main research interests are MANET and protocol simulation.

Zhi-mei Li, She received M. S. degree in Computer Science from Hunan university, changsha, China, in 2011. She is currently associate professor of Guilin university of Aerospace Technology, Guilin, China. Hers main research interests are Information technology and computer networks. 